\title{
Transcriptional activation: risky business
}

\author{
William P. Tansey \\ Cold Spring Harbor Laboratory, Cold Spring Harbor, New York 11724, USA
}

\begin{abstract}
Transcriptional regulation is all about getting RNA polymerase to the right place on the gene at the right time and making sure that it is competent to conduct transcription. Traditional views of this process place most of their emphasis on the events that precede initiation of transcription. We imagine a promoter-bound transcriptional activator (or collection of activators) recruiting components of the basal transcriptional machinery to the DNA, eventually leading to the recruitment of RNA polymerase II and the onset of gene transcription. Although these events play a crucial role in regulating gene expression, they are only half the story. Correct regulation of transcription requires that polymerase not only initiates when and where it should, but that it stops initiating when no longer appropriate. But how are the signals from transcriptional activators, telling RNA polymerase to fire, terminated? Is this process governed by chance, with activators simply falling off the promoter at a certain frequency? Or is there some more direct mechanism, whereby activators are aggressively limited from uncontrolled promoter activation? A new article by Chi et al. (2001) suggests the latter may be true, and provides a mechanism for how a component of the basal transcription machinery can mark the activators it has encountered, sentencing them to an early death or banishing them from the nucleus. The ability of the basal transcriptional apparatus to mark activators provides an efficient way to limit activator function and ensures that continuing transcription initiation at a promoter is coupled to the continuing synthesis and activation of transcriptional activators.
\end{abstract}

\section{Srb10 targets GCN4 for ubiquitin-mediated proteolysis}

In a nutshell, the Chi et al. (2001) study shows that phosphorylation of the transcription factor GCN4 by a kinase called Srb10 signals GCN4 destruction by ubiquitin (Ub)-mediated proteolysis. Although the finding that a kinase can regulate protein destruction is not extraordinary-most Ub-mediated proteolysis is regulated by phosphorylation (Hochstrasser 1996) — what is extraordinary about this story is the fact that Srb10 is also a component of the RNA polymerase II holoenzyme (Liao et al. 1995), suggesting that GCN4 is marked for destruction as a consequence of its ability to activate transcription.

To appreciate the significance of the Chi et al. (2001) findings, we need to know a little bit about the nature of GCN4 and of Srb10. GCN4 is a transcriptional activator that regulates amino acid biosynthesis in the yeast Saccharomyces cerevisiae. It is a basic helix-loop-helix leucine zipper transcription factor, belonging to the AP-1 family of transcriptional activators (Struhl 1987). Like many transcription factors, GCN4 expression and activity are subject to a tight series of controls that are exerted at the transcriptional (Albrecht et al. 1998), translational (Mueller and Hinnebusch 1986), and post-translational (Kornitzer et al. 1994) levels. Like many transcription

E-MAIL tansey@cshl.org; FAX (516) 367-8874. Article and publication are at www.genesdev.org/cgi/doi/10.1101/ gad.896501. factors, GCN4 is a highly unstable protein that is destroyed by Ub-mediated proteolysis (Kornitzer et al. 1994). And like many transcription factors, the expression and activity of GCN4 can be rapidly modulated to allow cells to mount quick and efficient responses to changes within their environment. When S. cerevisiae are subject to amino acid starvation, for example, GCN4 levels increase rapidly within the yeast cell. This transient increase in GCN4 levels-which is achieved as a result of increased GCN4 synthesis (Hinnebusch 1984; Albrecht et al. 1998) and decreased GCN4 destruction (Kornitzer et al. 1994)_results in a coordinated increase in the expression of $>30$ genes (Rolfes and Hinnebusch 1993), most of which encode enzymes for amino acid and purine biosynthesis. As a central player in this coordinated "general control" response, GCN4 allows yeast to reprogram their biosynthetic capabilities until their amino acid and nutrient needs can be met by the environment.

By virtue of its history and key role in the general control of amino acid biosynthesis, transcriptional activation by GCN4 has been studied extensively. GCN4 carries an acidic type activation domain (Hope and Struhl 1986) that interacts with components of the basal transcriptional apparatus, recruits these components to promoter DNA, and executes necessary chromatin remodeling steps required for efficient activation of GCN4 target genes. Like many activation domains, the activation domain of GCN4 can interact with an impressive 
array of basal transcription factors. Interactions have been reported between GCN4 and GAL11 (Lee et al. 1999), components of the SAGA chromatin remodeling complex (Utley et al. 1998), the TFIID complex (Drysdale et al. 1998), and the mediator complex (Drysdale et al. 1998), a collection of Srb proteins and other proteins that associate with RNA polymerase II to form the RNA polymerase II holoenzyme (Hengartner et al. 1995). These interactions all probably contribute to the ability of GCN4 to activate transcription. Importantly, however, in the Chi et al. (2001) study, it is the latter association with the Srb proteins, and Srb10 specifically, that lies at the heart of how GCN4 stability is regulated.

Srb10 was first identified as a suppressor of deletions within the carboxyl-terminal domain (CTD) of RNA polymerase II (Liao et al. 1995). As with all Srb proteins, Srb10 exists as part of the large multiprotein mediator complex that is associated with the RNA polymerase CTD, and that can mediate response of RNA polymerase to transcriptional activators (Hengartner et al. 1995). Srb10 has received considerable attention because it is not only a component of the holoenzyme, but also a cyclin-dependent kinase (CDK; Liao et al. 1995). Through associations with its cyclin partner, Srb11, the Srb10/11 complex-a homolog of the metazoan cdk8/cyclin c complex (Tassan et al. 1995)-phosphorylates several substrates, including transcriptional activators and RNA polymerase itself. The functional consequences of these Srb10-mediated phosphorylation events are profound but differ depending on the substrate protein. Phosphorylation of the transcription factor GAL4 by Srb10 stimulates the ability of GAL4 to activate transcription (Hirst et al. 1999). Phosphorylation of the RNA polymerase II CTD by Srb10 represses transcription of some genes by blocking the ability of RNA polymerase to enter promoter complexes. And now, as revealed in the Chi et al. (2001) study, phosphorylation of GCN4 by Srb10 results in an entirely different outcome-signaling the destruction of GCN4 by Ub-mediated proteolysis.

A reasonable model for how Srb10 regulates GCN4 destruction is presented in Figure 1. GCN4 contains five potential sites of phosphorylation by cyclin-dependent kinases such as Srb10. When GCN4 interacts with Srb10, probably in the context of the RNA polymerase II holoenzyme, some or all of these sites are phosphorylated. It is this phosphorylation-which is facilitated by the actions of another CDK, Pho85 (Meimoun et al. 2000) - that primes GCN4 for interaction with a Ub-ligase complex known as $\mathrm{SCF}^{\mathrm{Cdc} 4}$. The $\mathrm{SCF}^{\mathrm{Cdc} 4}$ complex belongs to the Skp-Cullin-F-box family of Ub-ligases, which consist of several core components-Skp1, Cdc53, Rbx-1, and Cdc34-associated with a unique specificity factor referred to as an F-box protein (Skowyra et al. 1997). For GCN4, genetic (Kornitzer et al. 1994; Meimoun et al. 2000) and biochemical (Chi et al. 2001) evidence show that the F-box protein is Cdc4, which has been linked to the ubiquitylation of several substrates in yeast, including the CDK inhibitors Sicl (Feldman et al. 1997) and Far1 (Henchoz et al. 1997), and the DNA replication control factor Cdc6 (Sanchez et al. 1999). Cdc4
A

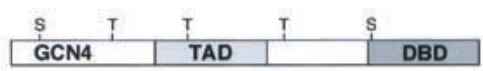

B

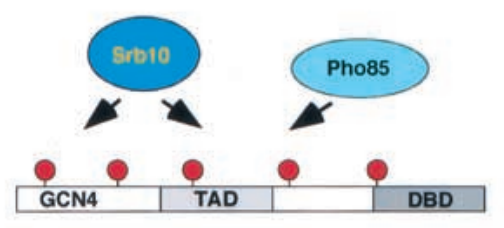

C

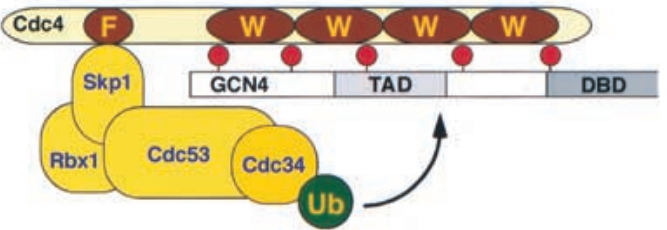

D

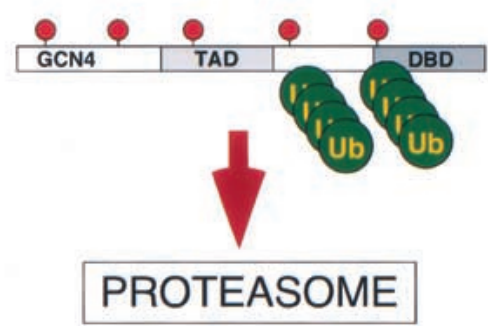

Figure 1. Srb10 phosphorylates GCN4 and signals its ubiquitylation by the SCF ${ }^{\mathrm{Cdc} 4} \mathrm{Ub}-$ ligase. $(A)$ The GCN4 protein contains five potential sites of phosphorylation ( $\mathrm{S}$ and $\mathrm{T}$ ) by cyclindependent kinases such as Srb10 and Pho85. (B) Interaction of GCN4 with Srb10 results in the phosphorylation of GCN4 (red circles). The Pho85 kinase also plays a role in promoting GCN4 phosphorylation, although the interplay between Srb10 and Pho85 is not known. $(C)$ Phosphorylated GCN4 is specifically recognized by the WD40 $(\mathrm{W})$ repeats within the F-box $(\mathrm{F})$ protein Cdc4. This recognition recruits GCN4 into the SCF ${ }^{\text {Cdc4 }} \mathrm{Ub}-$ ligase complex, which consists of Cdc4, Skp1, Rbx1, Cdc53, and the Ub-conjugating enzyme Cdc34. Cdc34, in turn, transfers Ub to (presumably) one or more lysine residues in GCN4, resulting in a highly ubiquitylated form of GCN4 $(D)$ that is rapidly destroyed by the $26 \mathrm{~S}$ proteasome. (DBD) DNA-binding domain; (TAD) transcriptional activation domain; $(\mathrm{Ub})$ ubiquitin.

contains two functionally relevant domains: an F box, that serves as a site for interaction with Skp1 and the rest of the SCF complex, and a series of WD40 repeats that mediate substrate recognition (Skowyra et al. 1997). It is presumably these repeats that specifically recognize phosphorylated GCN4, recruiting it into the $\mathrm{SCF}^{\mathrm{Cdc} 4}$ complex and allowing GCN4 to be ubiquitylated by the 
Cdc34 Ub-conjugating enzyme. Ubiquitylated GCN4 then is targeted to the $26 \mathrm{~S}$ proteasome, where it is broken down into small peptides and amino acids (Varshavsky 1997).

Although there are many factors that are involved in the destruction of GCN4 by Ub-mediated proteolysis, the key regulatory step in this process is the requirement for GCN4 phosphorylation. For it is this requirement that allows the RNA polymerase holoenzyme (through the Srb10 kinase) to ultimately control the metabolic fate of the GCN4 protein.

\section{A "black widow" model for transcriptional activation}

At one level, the idea that a component of the basal transcription apparatus would signal destruction of a transcriptional activator is counterintuitive. After all, regulatory and basal transcription factors work together to control the initiation of transcription: why should Srb10 act to destroy one of its closest collaborators? Although the Chi et al. (2001) study stops short of answering this intriguing question, it seems likely that Srb10 is acting to limit the capacity of GCN4 to control gene expression. Given the role of Srb10 as part of the mediator, and the demonstration that mutations within the transcriptional activation domain of GCN4 stabilize the protein (Kornitzer et al. 1994), it is reasonable to surmise that the Srb10-mediated destruction of GCN4 is intimately tied to the ability of GCN4 to activation transcription. Thus, by acting at the very heart of how GCN4 regulates transcription-through its interactions with the basal transcriptional machinery-Srb10 is able to specifically and efficiently terminate active GCN4 protein.

How is Srb10-mediated control of GCN4 used to regulate transcription? One possible explanation is that this control is used to limit associations between GCN4 and the basal transcriptional machinery that occur off the DNA. When potent transcriptional activators, like GCN4, are expressed at high levels, they can squelch (Gill and Ptashne 1988) transcriptional activation by sequestering basal transcription factors away from productive promoter complexes. Mark Ptashne predicted in 1988 (Ptashne 1988) that, because of this behavior, transcriptional activators would need to be controlled very tightly. What better way to control these factors than to empower the basal machinery with the ability to fight back, and to target GCN4 for destruction? In this scheme, Srb10 forms part of a surveillance machinery that recognizes GCN4 as a transcriptional activator, restricts its accumulation, and prevents the global disruption of transcriptional control that results from squelching.

Another fascinating possibility, however, and one that is suggested by Chi and colleagues, is that Srb10 marks GCN4 for destruction during the act of transcription activation, thus limiting the amount of time that GCN4 can productively occupy a promoter before it is destroyed. This idea leads to the notion of a "black widow" model of transcription activation, in which activators are destroyed as a direct consequence of recruiting the basal transcriptional machinery to a promoter. One version of this black widow model is depicted in Figure 2. In this model, a promoter-bound molecule of GCN4 recruits, through its transcriptional activation domain, the RNA polymerase II holoenzyme and other basal transcription factors. Recruitment of these factors not only results in productive initiation of transcription, but also in the Srb10-dependent phosphorylation of GCN4,
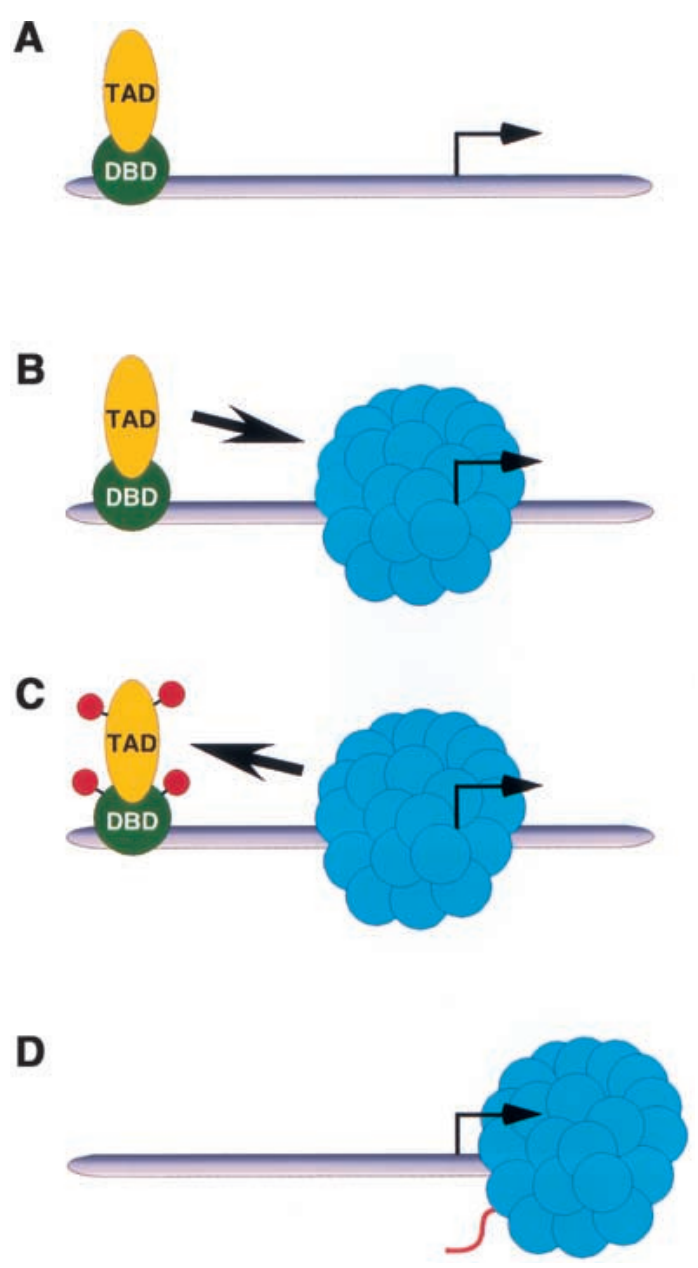

Figure 2. A black widow model of transcriptional activation. The Srb10-mediated destruction of GCN4, together with other evidence described in the text, supports a model in which the destruction of transcription factors like GCN4 is tied to their ability to activate transcription. In this model, a DNA-bound activator $(A)$, with its modular DNA-binding domain (DBD) and transcriptional activation domain (TAD), interacts with components of the basal transcriptional apparatus (shown in blue) and recruits these components to the promoter $(B)$, signaling transcription initiation. In turn, one or more of the basal factors marks the transcription factor by phosphorylation $(C)$. In the case of GCN4, it is the Srb10 component of the RNA polymerase holoenzyme that phosphorylates GCN4, leading to its ubiquitin-mediated destruction (see Fig. 1). In this way, the activator is cleared from the promoter $(D)$ preventing multiple rounds of transcriptional activation by a single promoter-bound transcription factor. 
which, in turn, signals GCN4 ubiquitylation and its destruction by the proteasome. The destruction of GCN4 after transcriptional stimulation provides an efficient way to prevent a single promoter-bound molecule of GCN4 from activating multiple rounds of transcription initiation and tightly links the function of GCN4 to other mechanisms that regulate its expression: in this scheme, activation of GCN4 target genes is tightly coupled to ongoing synthesis of the GCN4 protein, which (as discussed above) is a highly regulated process.

The concept that the basal transcription apparatus can mark activators it has encountered provides an attractive and logical way to control transcriptional activation and sheds light on an area of gene regulation that has been little studied in the past. Like all good stories, however, the Chi et al. (2001) study raises more questions than it answers. Does this process occur at all GCN4-regulated promoters? Does it require promoters at all? How does phosphorylated GCN4 encounter $\mathrm{SCF}^{\mathrm{Cdc} 4}$ within the nucleus? Is this a chance meeting? Or does a more direct association of the ubiquitylation and transcriptional machineries coordinate GCN4 destruction? Perhaps one of the most intriguing issues to be resolved at this point, however, is timing. When in the transcription cycle does Srb10 phosphorylate GCN4? Is it before GCN4 stimulates the initiation of transcription, or afterward? Understanding the timing of this event is crucial to understanding its significance to transcription. If Srb10 phosphorylates GCN4 before the initiation of transcription, it is possible that this phosphorylation may be required for transcriptional activation-as is observed for Srb10mediated phosphorylation of GAL4 (Hirst et al. 1999). In this way, by using the same signal (phosphorylation) to both activate GCN4 and to signal its destruction, the basal transcription machinery would maintain very tight control over GCN4, guaranteeing that only the labile form of GCN4 can activate gene expression. Alternatively, if Srb10 phosphorylates GCN4 after the initiation of transcription, it is possible that this phosphorylation serves to mark an activator as used, signaling its destruction before a second round of transcriptional activation can occur. Understanding how these events are timed, together with a better understanding of how Srb10-mediated regulation of GCN4 impacts on transcription, will begin to expose the true nature of how Srb10 is used to control GCN4 activity.

\section{All transcription factors should be regulated in this way}

Transcriptional regulation is one of the most highly conserved processes in biology. Throughout the broad spectrum of eukaryotic life, recurring themes in transcriptional control (underpinned by an extraordinary conservation of the transcriptional machinery) are used to regulate gene expression. Given the conservation of this process, therefore, it is difficult to imagine that control of transcriptional activators by the basal machinery is restricted to just GCN4 and just $S$. cerevisiae. Indeed, there is already an example of this type of regulation in metazoan cells. Vandel and colleagues (Vandel and Kouzarides 1999) have shown that the basal transcription factor kinase TFIIH phosphorylates residues within the E2F-1 activation domain, signaling destruction of E2F-1 by Ub-mediated proteolysis. Thus, there is a direct parallel between the control of GCN4 and E2F-1 by basal factor kinases, and it seems likely that other examples will surface in the near future.

In support of this concept, there is a wealth of circumstantial evidence suggesting that the process of transcriptional activation frequently is associated with transcription factor destruction. As a whole, transcription factors tend to be some of the most unstable proteins in the cell. Mammalian transcription factors such as E2F-1 (Hateboer et al. 1996), Jun (Treier et al. 1994), Fos (Tsurumi et al. 1995), p53 (Chowdary et al. 1994), Stat1 (Kim and Maniatis 1996), and Myc (Gross-Mesilaty et al. 1998), for example, are all short-lived proteins that are destroyed by Ub-mediated proteolysis. In most cases, the transcriptional activation domains of these proteins are required for their destruction (see Salghetti et al. [1999] for discussion), implying that it is the very ability of these proteins to activate transcription that signals their demise. This concept is further reinforced by the behavior of transcription factors such as microphthalmia $(\mathrm{Wu}$ et al. 2000), that is only destroyed in its transcriptionally active, and not inactive, state. Moreover, analysis of natural (Salghetti et al. 1999; Wang et al. 2000) and synthetic (Molinari et al. 1999; Salghetti et al. 2000) activation domains has shown that, at least for acidic-type activation domains, there is a close correlation between the ability of a transcription factor to activate transcription and the efficiency with which it is destroyed by Ub-mediated proteolysis. Although the molecular details of how most transcription factors are targeted for destruction is unknown, all of these observations point to a model in which interactions of transcription factors with the basal transcriptional apparatus leads to activator destruction. Perhaps Srb10 and its homologs (metazoan cdk8; Tassan et al. 1995) regulate the stability of most unstable transcription factors. Perhaps these responsibilities are shared between Srb10 and other kinases in the basal transcriptional machinery such as TFIIH (Lu et al. 1992) and $\mathrm{TAF}_{\mathrm{II}} 250$ (Dikstein et al. 1996). Or perhaps other catalytic functions within the basal apparatus, yet to be discovered, perform a similar regulatory role. Regardless of the details, however, it appears clear that the type of regulation suggested by the Chi et al. (2001) study is an important and recurring, theme in eukaryotic gene control.

Of course, not all transcription factors are unstable. Indeed, analysis of different types of transcriptional activation domains has revealed that the ability to signal Ub-mediated proteolysis is unique to domains rich in acidic residues-activation domains that are rich in proline or glutamine residues, for example, do not signal protein turnover (Salghetti et al. 2000). This finding suggests that it is the precise mechanism through which an activation domain stimulates RNA polymerase that determines whether or not a transcription factor will be 
destroyed. Does this mean that, by activating transcription in a certain way, some transcription factors can escape the kind of regulation described for Srb10 and GCN4? Not necessarily. An intriguing twist in the Chi et al. (2001) story is that Srb10 also can regulate the fate of a stable transcription factor, the multistress response protein Msn2 (Martinez-Pastor et al. 1996). At present, the molecular details are not fully understood, but it appears that Srb10 phosphorylates Msn2, promoting its rapid exclusion from the nucleus. Thus, although the stability of Msn2 is unaffected by phosphorylation, the net effect of Srb10-mediated phosphorylation is the same-to get Msn2 away from promoter DNA, away from the basal transcriptional machinery, and to, in turn, restrict the capacity of Msn2 to regulate transcription. In this circumstance, all of the regulatory consequences that have been discussed for GCN4 still apply, except that Msn2 is shunted out of the nucleus, rather than destroyed by Ub-mediated proteolysis. The Chi et al. (2001) study provides just a brief glimpse of this alternative form of transcription factor regulation, so a deeper understanding of the significance of Msn2 nuclear exclusion-and a deeper general knowledge of the mechanisms that regulate transcription factor localizationwill be needed before the broad significance of the Srb10-Msn2 interaction can be established.

\section{Conclusion}

The correct regulation of gene transcription is essential to the maintenance of normal cellular homeostasis. One of the main ways cells regulate gene expression is to maintain tight control over transcription factors, and mechanisms have been described that limit the abundance, distribution, and activity of these transcriptional regulators. The recent studies described here suggest that cells possess another weapon in their arsenal to regulate transcription - the ability of the basal transcriptional apparatus to mark the activators it encounters, sentencing them to an early death or banishing them to the antipodes. By deciding the fate of transcription factors at the point at which they function, cells maintain tight control over some of the most potent regulatory molecules in the cell. No doubt about it: Activating transcription is a risky business.

\section{References}

Albrecht, G., Mosch, H.U., Hoffmann, B., Reusser, U., and Braus, G.H. 1998. Monitoring the Gen4 protein-mediated response in the yeast Saccharomyces cerevisiae. J. Biol. Chem. 273: 12696-12702.

Chi, Y., Huddleston, M.J., Zhang, X., Young, R.A., Annan, R.S., Carr, S.A., and Deshaies, R.J. 2001. Negative regulation of Gcn 4 and Msn2 transcription factors by Srb10 cyclin-dependent kinase. Genes \& Dev. 15: 1078-1092.

Chowdary, D.R., Dermody, J.J., Jha, K.K., and Ozer, H.L. 1994. Accumulation of p53 in a mutant cell line defective in the ubiquitin pathway. Mol. Cell. Biol. 14: 1997-2003.

Dikstein, R., Ruppert, S., and Tjian, R. 1996. TAFII250 is a bipartite protein kinase that phosphorylates the base tran- scription factor RAP74. Cell 84: 781-790.

Drysdale, C.M., Jackson, B.M., McVeigh, R., Klebanow, E.R., Bai, Y., Kokubo, T., Swanson, M., Nakatani, Y., Weil, P.A., and Hinnebusch, A.G. 1998. The Gcn4p activation domain interacts specifically in vitro with RNA polymerase II holoenzyme, TFIID, and the Adap-Gen5p coactivator complex. Mol. Cell. Biol. 18: 1711-1724.

Feldman, R.M., Correll, C.C., Kaplan, K.B., and Deshaies, R.J. 1997. A complex of Cdc4p, Skp1p, and Cdc53p/cullin catalyzes ubiquitination of the phosphorylated CDK inhibitor Sic1p. Cell 91: 221-230.

Gill, G. and Ptashne, M. 1988. Negative effect of the transcriptional activator GAL4. Nature 334: 721-724.

Gross-Mesilaty, S., Reinstein, E., Bercovich, B., Tobias, K.E., Schwartz, A.L., Kahana, C., and Ciechanover, A. 1998. Basal and human papillomavirus E6 oncoprotein-induced degradation of Myc proteins by the ubiquitin pathway. Proc. Natl. Acad. Sci. 95: 8058-8063.

Hateboer, G., Kerkhoven, R.M., Shvarts, A., Bernards, R., and Beijersbergen, R.L. 1996. Degradation of E2F by the ubiquitin-proteasome pathway: Regulation by retinoblastoma family proteins and adenovirus transforming proteins. Genes \& Dev. 10: 2960-2970.

Henchoz, S., Chi, Y., Catarin, B., Herskowitz, I., Deshaies, R.J., and Peter, M. 1997. Phosphorylation- and ubiquitin-dependent degradation of the cyclin-dependent kinase inhibitor Farlp in budding yeast. Genes \& Dev. 11: 3046-3060.

Hengartner, C.J., Thompson, C.M., Zhang, J., Chao, D.M., Liao, S.M., Koleske, A.J., Okamura, S., and Young, R.A. 1995. Association of an activator with an RNA polymerase II holoenzyme. Genes \& Dev. 9: 897-910.

Hinnebusch, A.G. 1984. Evidence for translational regulation of the activator of general amino acid control in yeast. Proc. Natl. Acad. Sci. 81: 6442-6446.

Hirst, M., Kobor, M.S., Kuriakose, N., Greenblatt, J., and Sadowski, I. 1999. GAL4 is regulated by the RNA polymerase II holoenzyme-associated cyclin-dependent protein kinase SRB10/CDK8. Mol. Cell 3: 673-678.

Hochstrasser, M. 1996. Ubiquitin-dependent protein degradation. Annu. Rev. Genet. 30: 405-439.

Hope, I.A. and Struhl, K. 1986. Functional dissection of a eukaryotic transcriptional activator protein, GCN4 of yeast. Cell 46: 885-894.

Kim, T.K. and Maniatis, T. 1996. Regulation of interferon- $\gamma-$ activated STAT1 by the ubiquitin-proteasome pathway. Science 273: 1717-1719.

Kornitzer, D., Raboy, B., Kulka, R.G., and Fink, G.R. 1994. Regulated degradation of the transcription factor Gen4. EMBO J. 13: 6021-6030.

Lee, Y.C., Park, J.M., Min, S., Han, S.J., and Kim, Y.J. 1999. An activator binding module of yeast RNA polymerase II holoenzyme. Mol. Cell. Biol. 19: 2967-2976.

Liao, S.M., Zhang, J., Jeffery, D.A., Koleske, A.J., Thompson, C.M., Chao, D.M., Viljoen, M., van Vuuren, H.J., and Young, R.A. 1995. A kinase-cyclin pair in the RNA polymerase II holoenzyme. Nature 374: 193-196.

Lu, H., Zawel, L., Fisher, L., Egly, J.M., and Reinberg, D. 1992. Human general transcription factor IIH phosphorylates the C-terminal domain of RNA polymerase II. Nature 358: 641645.

Martinez-Pastor, M.T., Marchler, G., Schuller, C., MarchlerBauer, A., Ruis, H., and Estruch, F. 1996. The Saccharomyces cerevisiae zinc finger proteins Msn2p and Msn4p are required for transcriptional induction through the stress response element (STRE). EMBO J. 15: 2227-2235.

Meimoun, A., Holtzman, T., Weissman, Z., McBride, H.J., Still- 
man, D.J., Fink, G.R., and Kornitzer, D. 2000. Degradation of the transcription factor Gcn 4 requires the kinase Pho85 and the SCF(CDC4) ubiquitin-ligase complex. Mol. Biol. Cell 11: 915-927.

Molinari, E., Gilman, M., and Natesan, S. 1999. Proteasomemediated degradation of transcriptional activators correlates with activation domain potency in vivo. EMBO J. 18: 64396447.

Mueller, P.P. and Hinnebusch, A.G. 1986. Multiple upstream AUG codons mediate translational control of GCN4. Cell 45: 201-207.

Ptashne, M. 1988. How eukaryotic transcriptional activators work. Nature 335: 683-689.

Rolfes, R.J. and Hinnebusch, A.G. 1993. Translation of the yeast transcriptional activator GCN4 is stimulated by purine limitation: Implications for activation of the protein kinase GCN2. Mol. Cell. Biol. 13: 5099-5111.

Salghetti, S.E., Kim, S.Y., and Tansey, W.P. 1999. Destruction of Myc by ubiquitin-mediated proteolysis: Cancer-associated and transforming mutations stabilize Myc. EMBO J. 18: 717-726.

Salghetti, S.E., Muratani, M., Wijnen, H., Futcher, B., and Tansey, W.P. 2000. Functional overlap of sequences that activate transcription and signal ubiquitin-mediated proteolysis. Proc. Nat1. Acad. Sci. 97: 3118-3123.

Sanchez, M., Calzada, A., and Bueno, A. 1999. The Cdc6 protein is ubiquitinated in vivo for proteolysis in Saccharomyces cerevisiae. J. Biol. Chem. 274: 9092-9097.

Skowyra, D., Craig, K.L., Tyers, M., Elledge, S.J., and Harper, J.W. 1997. F-box proteins are receptors that recruit phosphorylated substrates to the SCF ubiquitin-ligase complex [see comments]. Cell 91: 209-219.

Struhl, K. 1987. The DNA-binding domains of the jun oncoprotein and the yeast GCN4 transcriptional activator protein are functionally homologous. Cell 50: 841-846.

Tassan, J.P., Jaquenoud, M., Leopold, P., Schultz, S.J., and Nigg, E.A. 1995. Identification of human cyclin-dependent kinase 8 , a putative protein kinase partner for cyclin C. Proc. Natl. Acad. Sci. 92: 8871-8875.

Treier, M., Staszewski, L.M., and Bohmann, D. 1994. Ubiquitindependent c-Jun degradation in vivo is mediated by the delta domain. Cell 78: 787-798.

Tsurumi, C., Ishida, N., Tamura, T., Kakizuka, A., Nishida, E., Okumura, E., Kishimoto, T., Inagaki, M., Okazaki, K., Sagata, N., et al. 1995. Degradation of c-Fos by the 26S proteasome is accelerated by c-Jun and multiple protein kinases. Mol. Cell. Biol. 15: 5682-5687.

Utley, R.T., Ikeda, K., Grant, P.A., Cote, J., Steger, D.J., Eberharter, A., John, S., and Workman, J.L. 1998. Transcriptional activators direct histone acetyltransferase complexes to nucleosomes. Nature 394: 498-502.

Vandel, L. and Kouzarides, T. 1999. Residues phosphorylated by TFIIH are required for E2F-1 degradation during S-phase. EMBO J. 18: 4280-4291.

Varshavsky, A. 1997. The ubiquitin system. Trends Biochem. Sci. 22: 383-387.

Wang, D., Moriggl, R., Stravopodis, D., Carpino, N., Marine, J.C., Teglund, S., Feng, J., and Ihle, J.N. 2000. A small amphipathic $\alpha$-helical region is required for transcriptional activities and proteasome-dependent turnover of the tyrosinephosphorylated Stat5. EMBO J. 19: 392-399.

Wu, M., Hemesath, T.J., Takemoto, C.M., Horstmann, M.A., Wells, A.G., Price, E.R., Fisher, D.Z., and Fisher, D.E. 2000. c-Kit triggers dual phosphorylations, which couple activation and degradation of the essential melanocyte factor microphthalmia. Genes \& Dev. 14: 301-312. 


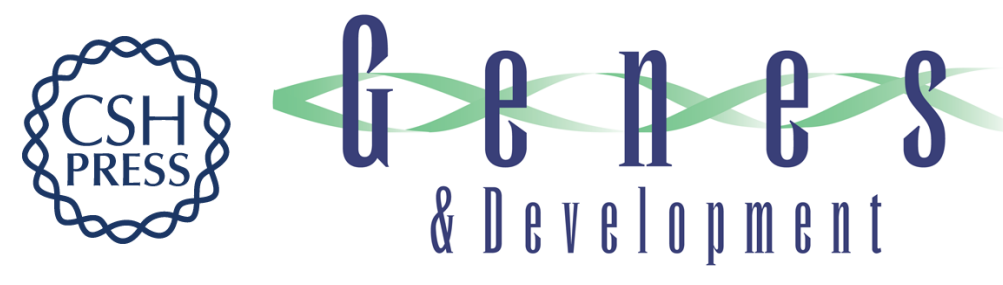

\section{Transcriptional activation: risky business}

William P. Tansey

Genes Dev. 2001, 15:

Access the most recent version at doi:10.1101/gad.896501

References This article cites 39 articles, 21 of which can be accessed free at: http://genesdev.cshlp.org/content/15/9/1045.full.html\#ref-list-1

License

Email Alerting Receive free email alerts when new articles cite this article - sign up in the box at the top Service right corner of the article or click here.

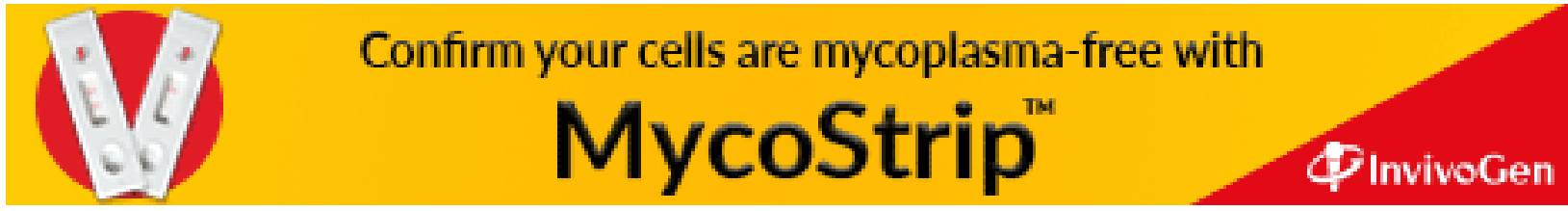

